\title{
PLACENTAL DYSFUNCTION: HEALTH STATUS, NUTRITIONAL STATUS AND MINERAL PROFILE OF A MOTHER-CHILD PAIR
}

DOI: 10.36740/WLek202001118

\author{
Tetyana V. Frolova', Viktoriya V. Lazurenko², Nana M. Pasiyeshvili ${ }^{3}$ Anastasiia G. Amash', Yevhen Y. Bilyi ${ }^{2}$, \\ Nataliya F. Stenkova ${ }^{1}$ \\ 'DEPARTMENT OF PROPEDEUTIC PEDIATRICS No. 1, KHARKIV NATIONAL MEDICAL UNIVERSITY, KHARKIV, UKRAINE \\ 2DEPARTMENT OF OBSTETRICS AND GYNECOLOGY No. 2, KHARKIV NATIONAL MEDICAL UNIVERSITY, KHARKIV, UKRAINE \\ ${ }^{3}$ KHARKIV REGIONAL CLINICAL PERINATAL CENTER, KHARKIV, UKRAINE
}

\begin{abstract}
The aim: the analysis of the PD, pregnancy, the labor, the research on peculiarities of the development and health status of breast-fed children, who are born to mothers with PD, by means of analyzing the mother-child pair's nutritional status and mineral homeostasis.

Materials and methods: At the 1 stage, an analysis of the PD frequency, the pregnancy, the labor was conducted during 5 years. At the 2 stage, 188 mother-child pairs were examined: $84.04 \%$ women had PD and $15.96 \%$ didn't have it. The research included the analysis of the anamnestic data, maternal nutritional status, general clinical study, assessment of the physical, psychomotor level of the child's development, study of the elemental profile.

Results: High frequency of complications in pregnancy and labor was observed in cases when women had PD, due to the imbalance in the "mother-placenta-fetus" system. The results' analysis showed an increased level of $\mathrm{Zn}(1.437 \%), \mathrm{K}(10.147 \%)$, and $\mathrm{Ca}(83.900 \%)$ in hair; an increased level of K (82.818\%), Cr (0.274\%), and $\mathrm{Na}$ (3.611\%) in breast milk of women with PD. Children born to mothers with PD had a significantly increased level of $\mathrm{Cr}(0.92 \%), \mathrm{S}(0.578 \%)$ and P (0.169\%), Na (0.107\%), Ca (56.041\%), and Zn (7.149\%). Conclusions: PD has a negative impact on the pregnancy and labor and may be one of the factors causing the mineral imbalance of breast-fed infant.
\end{abstract}

KEY WORDS: mother-child pair, placental dysfunction, state of health, nutritional status, mineral profile

Wiad Lek. 2020;73(1):95-98

\section{INTRODUCTION}

At present, the issue of studying the medical and social risk factors in terms of developing interdependent disorders in a mother-child pair's state of health is raised in separate works, which only fragmentarily show the essence of the problem concerning the effects of pregnancy complications on the child's health. The most pressing issue of modern obstetrics is the placental dysfunction (PD), since it is the main cause of complications during the pregnancy and labor, it greatly increases the incidence of perinatal morbidity and mortality, leads to complications in the postnatal period, fosters disability cases of a newborn child. PD can promote fetal programming to develop adverse health consequences in childhood $[1,2]$. According to recent studies, during the pregnancy external factors, including microbial agents, significantly affect the fetal-placental complex and cause the development of newborns' health complications that allows the placenta to be considered as the primary route of prenatal transmission of damaging factors from mother to fetus [3]. If the fetus survives, the child may have an increased risk of morbidity throughout the life [4]. At present, the effect of a mother's nutrition and her past diseases on the placenta development has been proved [5]. That is why ensuring the adequate nutritional status of women during pregnancy is one of the essential and obligatory conditions for the normal placenta functioning and, accordingly, the birth of a healthy child.

Thus, the study of the health of women, as well as of children born to mothers with placental dysfunction and PD impact on the further development of the child, is an urgent issue that caused the conduct of this research.

\section{THE AIM}

The aim is to conduct a retrospective analysis of the PD cases, pregnancy course, the labor and the state of newborns, the research on peculiarities of the development and health status of children that are born to mothers with placental dysfunction and are exclusively breast-fed, by means of analyzing the mother-child pair's nutritional status and mineral homeostasis

\section{MATERIALS AND METHODS}

At the first stage, a retrospective analysis of the PD frequency, the course of pregnancy, the labor and the state of newborns during 5 years (examining 14276 women) was conducted to analyze the prevalence of this pathology.

To clarify the mechanisms of PD development and the development of clinical and pathogenetic approaches to 
its prevention, 188 "mother-child" pairs were examined. The experimental group consisted of 158 (84.04\%) mother-child pairs with mothers having the PD; the control group - $30(15.96 \%)$ pairs with mothers having uncomplicated pregnancy.

The general clinical study of pregnant women was conducted in accordance with the Order of the Ministry of Health of Ukraine No. 503 of 28.12.2002 [6].

All pregnant women, in addition to the general clinical and laboratory examination, underwent dynamic ultrasound and doplerometric examinations, a cardiotocography (CTG), and examinations to determine the biophysical profile of the fetus (BPP). To interpret cardiotocograms, a point rating system by W. Fisher (1976), that was later improved by I. O. Makarov, was used [7]. Prenatal screening was conducted to determine the possible risk of birth of a child with congenital malformations; screening included the identification of biochemical, ultrasound markers of chromosomal pathology during 11-13 weeks, geneticist's counseling at 20th-22nd week. In addition, the identification of women's mineral profile during pregnancy and lactation was conducted by determining the relatively toxic (Al, Ti, Ba, Pb, Sr) and essential (Fe, I, C, Zn, Co, Cr, Mo, $\mathrm{Ni}, \mathrm{V}$, Se, Mn, As, F, Si, Br and Li) elements in samples of hair and breast milk by spectrometry method using the ElvaxLight apparatus (Ukraine, 2008). Hair was chosen as a biological material for research, since it reflects the long exposure of the elements in a human body.

The second stage of the research involved examining a "mother-child" pair when the child was 5 months of age, namely, before the introduction of complementary feeding, and it included, in addition to analysis of anamnestic data, general clinical examination with an assessment of the level of physical and psychomotor development of the child, ME study: for children - using the hair; for mothers - using the hair and breast milk to determine the mineral profile of a "mother - child" pair.

Statistical processing of the obtained results was carried out by methods of mathematical statistics. For each statistic series, the arithmetic mean (M) was calculated as well as the mean error of the arithmetic mean $(\mathrm{m})$. The estimation of the probability of differences in average mean in groups ( $p$ ) was calculated using Student's criterion ( $t$ ). The difference was considered probable if a value of $p<0.05$. Statistical processing of the obtained research results was performed on a personal computer using Microsoft Excel program.

\section{RESULTS AND DISCUSSION}

According to a retrospective analysis, over the past 5 years the frequency of PD among pregnant women has increased from $5.91 \%$ to $15.35 \%$. In order to determine the possible influence of PD on the incidence rate among newborns, it is necessary to understand the structure of morbidity of mature and premature infants. According to the results of the retrospective analysis based on the work of the city's perinatal center, over the past 5 years the number of the fetal growth restriction syndrome (FGR), caused by PD in infant newborns (14.8\%), has increased significantly compared with its number 5 years ago (5.8\%). The incidence of congenital pneumonia increased from $1.2 \%$ to $3.4 \%$, the incidence of intrauterine infection - from $1.7 \%$ to $2.02 \%$. Among premature infants the incidence of intrauterine infection increased from $10.4 \%$ to $15.7 \%$, FGR frequency increased from $7.3 \%$ to $12.5 \%$. The main causes of intrauterine fetal death were: chronic fetoplacental insufficiency caused by infectious lesions, preeclampsia, extragenital pathology; congenital malformations of the fetus, umbilical-placental circulation disorders. The placental dysfunction has led to FGR and subsequent fetal death in $30.3 \%$ of cases. Conducting histological examination of placenta, markers of placental dysfunction were detected in $50.2 \%$ of cases, infectious lesions (exudative villusitis, pyogenic chorioamnionitis) were detected in $66.7 \%$ of cases. It is morphologically proved that PD leads to perivascular hemorrhages, angiomatosis, pyogenic necrotic deciduitis, funiculitis, or is a consequence of perinatal infections [8].

The results analysis of the examination of the "mother-child" pairs showed that the pregnant women in the experimental group were from 16 to 42 years of age that amounted to an average of $28.4 \pm 5.2$ years old. In the control group, the pregnant were $22.6 \pm 4.5$ years old ranging from 18 to 36 years old. A significant proportion of pregnant women, who are over 30, draws the attention: in the experimental group $-39.2 \%$ of women, whereas in the control group $-16.7 \%$. The ratio of resident of the city and the country in the experimental and the control groups was unlikely to differ ( $p>0.05$ ). The largest part in both groups was represented by housewives, which may be due to socio-economic conditions that have developed recently in Ukraine. Living conditions of the examined women were satisfactory, 16 women of the experimental $(10.1 \%)$ group smoked before the pregnancy, 2 (1.3\%) - during the pregnancy. Alcoholic beverages were not abused. Anemia of varying severity was observed in 23 (14.6\%) women in the experimental group, while in the control group, it was observed in $2(6.6 \%)$ women, which could be considered as one of the causes of PD in pregnancy.

Among women of the experimental and the control groups, the primiparous ones were $79(45.6 \%)$ and $12(40 \%)$ women, which indicates the comparability of the given groups. Analyzing the previous pregnancy courses of the primiparas, it was determined that the previous pregnancies of women in the experimental group ended mostly in spontaneous or artificial abortions, missed miscarriages.

During doplerometric examination of uterine arteries, an increase in vascular resistance was observed in $70.9 \%$ of pregnant women with PD. Quantitative evaluation of blood flow in the umbilical artery showed that the amount of the pulsation index during the second and third trimesters varies from 1.8 to 2.10. Similar data were found in dopplerometric results of blood flow of the mesencephalic artery and fetal aorta. Thus, the dopplerometric study clearly demonstrates the negative changes in the blood flow in the mother-placenta-fetus system of pregnant women with PD.

On measuring the BPP, attention was drawn to non- 
stress tests, breathing movements, fetal motor activity, tonus, amniotic index and placental maturity degree. The last two indicators characterize the degree of the PD. Rate of the BPP using the scale by A. Vintzileos (1987) ranged from $8.8 \pm 0.2$ points in the experimental group to $11.9 \pm$ 0.1 points in the control group $(\mathrm{p}<0.05)$.

Comparative analysis of fetal CTG of pregnant women with PD showed severe disorders of the fetus in $11.4 \%$ of cases and moderate ones in $26.6 \%$. It should be noted that $142(89.9 \%)$ women in the experimental group had infectious inflammatory diseases of the reproductive system, diagnosis of which was carried out on the basis of complaints, clinical picture, laboratory test results.

Among the pathogens staphylococcus, streptococcus, enterococcus, coli-bacillary infection, herpes causative agents, gardnerellosis, toxoplasmosis prevailed. The analysis of pregnancy course of women with PD showed the following complications: vomiting of pregnant women $-36.7 \%$, threatened miscarriage $-30.4 \%$, the threat of preterm labor - $45.6 \%$, preeclampsia - $22.8 \%$, fetal distress - $18.4 \%$. Labor analysis showed that preterm labor occurred in $13.9 \%$ of the pregnant women in the experimental group, fetal distress $-51.3 \%$, premature rupture of fetal membranes $9.5 \%$. Methods of labour: in $84.2 \%$ of cases - vaginal birth, $15.8 \%$ - cesarean section. The fetal state was assessed to 8 points and above according to the Apgar score in $70.9 \%$ of cases. In other words, the analysis of pregnancy course and labor among women with PD showed a high incidence of complications of pregnancy and labor, which is caused by the imbalance in the mother-placenta-fetus system, which coincides with the data of other researchers [9].

The results of the maternal nutrition questionnaire using the Dietplan-6 program (https://dietplan6.software. informer.com/6.6/) showed that $60.1 \%$ of women with $\mathrm{PD}$ had a lack of calcium, iron, magnesium and protein in their diet , and 30,2\% - lack of vitamins B6, B12, E, A and $\mathrm{D}, 58,6 \%$ - iodine deficiency. Among mothers who did not have a PD in their medical history, an imbalance of the diet was observed much less frequently: $20.1 \%$ of women had reduced calcium, magnesium, iron consumption; $5.6 \%$ had a lack of protein, vitamin C, fats; $15.2 \%$ - of iodine, vitamins $\mathrm{D}, \mathrm{B} 6$ and $\mathrm{B} 12$.

The result analysis of the mineral profile research of mothers with $\mathrm{PD}$ showed ME imbalance due to an increase in levels of $\mathrm{Zn}(1.437 \%, \mathrm{p} \leq 0.005), \mathrm{K}(10.147 \%, \mathrm{p} \leq 0.005)$, and a decrease in $\mathrm{Ca}(83.900 \%, \mathrm{p} \leq 0.005)$ level, whereas the mothers who did not have PD, had the level of $\mathrm{Zn} / \mathrm{K} /$ Ca within the normal range $(0.6667 \%$ / 9.133\% / 84.913\%, $\mathrm{p} \leq 0.005$ ).

A noteworthy aspect was that in hair samples of mothers with PD there was a decrease in levels of $\mathrm{Cr}(0.083 \%, \mathrm{p} \leq$ $0.005)$ and $\mathrm{Na}(1.820 \%, \mathrm{p} \leq 0.005)$, in contrast to hair samples of mothers without $\mathrm{PD}$, where only Cr level decrease is observed ( $0.114 \%, \mathrm{p} \leq 0.005)$. According to the results of breast milk spectrometry, it was determined that samples of the mothers with PD showed increased levels of $\mathrm{K}$ in $82.818 \%$ of cases, $\mathrm{Cr}-0.274 \%, \mathrm{Na}-3.611 \%$ (respectively, $\mathrm{p} \leq 0.005$ ), and samples of the mothers without PD showed an increase in levels of $\mathrm{K}$ in $80.631 \%$ of cases, $\mathrm{Cr}-0.251 \%$, $\mathrm{Na}-2.529 \%$ (respectively, $\mathrm{p} \leq 0.005$ ).

It should be noted that, the allegedly toxic ME: $\mathrm{Ti}$ $(0,043 \%, \mathrm{p} \leq 0,005), \mathrm{Sr}(0,068 \%, \mathrm{p} \leq 0,005)$ and toxic $\mathrm{Pb}$ $(0,002 \%, \mathrm{p} \leq 0,005)$ were detected in the hair of mothers of both groups. $\mathrm{Ti}(0.024 \%, \mathrm{p} \leq 0.005)$ and $\mathrm{Pb}(0.006 \%$, $\mathrm{p}$ $\leq 0.005$ ) were detected in the breast milk of the mothers of both groups,.

Anamnesis analysis of children born to mothers with PD showed that $16.4 \%$ of children had the manifestation of atopic dermatitis, 9.1\% had several cases of acute obstructive bronchitis, 5.3\% had laryngotracheitis, $15.5 \%$ had rhinopharyngitis, $8.0 \%$ - hip dysplasia. In a group of children born to women without PD, $4.2 \%$ of children had manifestation of atopic dermatitis and 1,3\% - hip dysplasia.

Assessing the level of physical development of the children, it was found that $3.7 \%$ of the children whose mothers had a medical history of PD, had a debilitation. 12.76\% of children born to the mothers, who did not have a PD during the pregnancy, were diagnosed to be prone to possible overweight. Neuropsychic development of children of both groups was up to the age. As to anamnestic data, $8.3 \%$ of children born to mothers with PD were known to have hypoxic-ischemic central nervous system (CNS) injury.

The results analysis of hair spectrometry showed that children born to mothers with and without PD didn't have significant differences in the levels of $\mathrm{Mg}, \mathrm{S}, \mathrm{Cl}, \mathrm{Fe}, \mathrm{Cu}, \mathrm{Ni}$, $\mathrm{Sr}, \mathrm{Pb}, \mathrm{Br}, \mathrm{Ci}$, Si and Mo.

At the same time, it was determined that the children born to mothers with PD had the increased content of $\mathrm{Cr}$ $-0.928 \%(\mathrm{p} \leq 0.005), \mathrm{S}-0.578 \%(\mathrm{p} \leq 0.005)$ and $\mathrm{P}-0.169 \%$ $(\mathrm{p} \leq 0.005)$ in comparison to the same indicators of the children born to mothers without PD: $\mathrm{S}-0.462 \%$ ( $\mathrm{p} \leq$ $0.005), \mathrm{Cr}-0.381 \%(\mathrm{p} \leq 0.005), \mathrm{P}-0.016 \%(\mathrm{p} \leq 0.005)$. It was also determined that among children born to mothers with PD there was an increase in levels of $\mathrm{Na}-0.107 \%(\mathrm{p}$ $\leq 0.005), \mathrm{Ca}-56.041 \%(\mathrm{p} \leq 0.005)$ and $\mathrm{Zn}-7.142 \%(\mathrm{p} \leq$ 0.005 ), which may be connected both with their alimentary intake and the influence of the environment $[10,11]$.

Among children born to women who did not have PD, there were no significant variations in $\mathrm{ME}$ values: $\mathrm{Na}$ (0.040\%), Ca (51.543\%), Zn (4.348\%), ( $\mathrm{p} \leq 0.005)$.

\section{CONCLUSIONS}

1. Placental dysfunction, caused predominantly by intrauterine infectious lesions of placenta, has a negative impact on the course of pregnancy and labor, the state of the fetus, newborn and infant.

2. Mother's placental dysfunction can be one of the factors of imbalance in mineral homeostasis of children aged up to 5 months, which are exclusively breast-fed.

3. The mineral profile of children, who are exclusively breast-fed, depends to a large extent on the nutritional pattern of the mother's diet, both during pregnancy and lactation.

4. Imbalance in mineral homeostasis of children born to mothers with PD, namely, the increase in levels of Zn, 
$\mathrm{Na}, \mathrm{Cr}, \mathrm{S}, \mathrm{P}$ and $\mathrm{Ca}$, may cause frequent respiratory diseases, a liability to allergic reactions and a disorder of the physical development level in the future.

5. The balance of women's nutrition during pregnancy and lactation should be considered as a means of preventing the mineral imbalance in children.

\section{REFERENCES}

1. Latendresse G., Founds S. J. Midwifery Womens Health, 2015 JulAug;60(4):360-70. doi: 10.1111/jmwh.12344. The Fascinatingand Complex RoleofthePlacenta in Pregnancy and Fetal Well-being.

2. Cuffe J.S.M., Holland O., Salomon C., Rice G.E., Perkins A.V. Placenta. 2017 Jun;54:104-110. doi: 10.1016/j.placenta.2017.01.119. Epub 2017 Jan 16. Review

3. Dindar 0.A. Diagnostic determinants of the fetoplacental complex functional state of the pregnant women with obesity and metabolic syndrome .Health of Woman. 2016;2: 90-94.

4. Sliusar T.Y .; Pylypenko, O.N .; Dzhelomanova, 0.A .; Levchenko, Y. I. Changes in the parameters of the fetoplacental complex of patients with fetal growth restriction and intrauterine fetal demise in medical history. Health of Woman.2015; 3: 156-158.

5. Zhang S., Regnault T.R., Barker P.L., Botting K.J., Mc Millen I.C., Mc Millan C.M., Roberts C.T., Morrison J.L. Nutrients. 2015 Jan 8;7(1):360-89. doi: $10.3390 /$ nu7010360. Placental adaptations in growth restriction.

6. Order of the Ministry of Health of Ukraine No. 503 of 28.12.2002

7. Makarov I.0., Yudina E.V. Cardiotocography during pregnancy and labor: a training manual.M.: Medpress-inform.2016; 45-55.

8. Vakoliuk A.V. The fetoplacental insufficiency syndrome in the pathology of pregnancy. Clinical and morphological aspects/ «Hyst»: The Ukrainian Student Medical Journal. 2015;17:256

9. Mykuliak Kh.V. Parada A.I. Features of the course of pregnancy and labor during fetoplacental dysfunction. «Hyst»: The Ukrainian Student Medical Journal;2018:20-21
10. Roohani, Nazanin, etal. "Zinc and its importance for human health: An integrative review." Journal of research in medical sciences: the official journal of Isfahan University of Medical Sciences 18.2 (2013): 144.

11. Larson, Nicole I., et al. "Calcium and dairy intakes of adolescents are associated with their home environment, taste preferences, personal health beliefs, and meal patterns." Journal of the American Dietetic Association 106.11 (2006): 1816-1824.

\section{ORCID and contributionship:}

Tetyana V. Frolova - 0000-0002-6861-8902 ${ }^{A, F}$

Viktoriya V. Lazurenko - 0000-0002-7300-4868 ${ }^{A}$

Nana M. Pasiyeshvili - 0000-0002-9340-7390 ${ }^{E}$

Anastasiia G. Amash - 0000-0001-5510-100X B,C,D

Yevhen Y. Bilyi - 0000-0002-3383-7088 B,C,D

Nataliya F. Stenkova - 0000-0002-7784-6747 ${ }^{E}$

\section{Conflict of interest:}

The Authors declare no conflict of interest

\section{CORRESPONDING AUTHOR Anastasiia G. Amash}

Borzenko str., h.7, app. 37, 61177, Kharkiv, Ukraine

e-mail:anastasiyaamash@gmail.com

Received: 21.04 .2019

Accepted: 11.11 .2019

A - Work concept and design, B - Data collection and analysis, C - Responsibility for statistical analysis, $\mathbf{D}$-Writing the article, $\mathbf{E}$-Critical review, $\mathbf{F}$ - Final approval of the article 